\title{
Radar Sensing for Activity Classification in Elderly People Exploiting Micro-Doppler Signatures Using Machine Learning
}

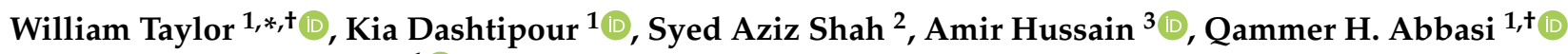 \\ and Muhammad A. Imran ${ }^{1}$ (D) \\ 1 James Watt School of Engineering, University of Glasgow, Glasgow G12 8QQ, UK; \\ kia.dashtipour@glasgow.ac.uk (K.D.); Qammer.Abbasi@glasgow.ac.uk (Q.H.A.); \\ Muhammad.Imran@glasgow.ac.uk (M.A.I.) \\ 2 Mobile Health, Centre of Intelligent Healthcare, Coventry University, Coventry CV1 5RW, UK; \\ syed.shah@coventry.ac.uk \\ 3 School of Computing, Edinburgh Napier University, Scotland EH10 5DT, UK; A.Hussain@napier.ac.uk \\ * Correspondence: 2536400t@student.gla.ac.uk \\ + These authors contributed equally to this work.
}

Citation: Taylor, W.; Dashtipour, K.; Syed, S.A.; Hussain, A.; Abbasi, Q.A.; Imran, M.A. Radar Sensing for Activity Classification in Elderly People Exploiting Micro-Doppler Signatures Using Machine Learning. Sensors 2021, 21, 3881. https:// doi.org/10.3390/s21113881

Academic Editor: Paolo Barsocchi

Received: 10 May 2021

Accepted: 2 June 2021

Published: 4 June 2021

Publisher's Note: MDPI stays neutral with regard to jurisdictional claims in published maps and institutional affiliations.

Copyright: (c) 2021 by the authors. Licensee MDPI, Basel, Switzerland. This article is an open access article distributed under the terms and conditions of the Creative Commons Attribution (CC BY) license (https:// creativecommons.org/licenses/by/ $4.0 /)$.

\begin{abstract}
The health status of an elderly person can be identified by examining the additive effects of aging along with disease linked to it and can lead to 'unstable incapacity'. This health status is determined by the apparent decline of independence in activities of daily living (ADLs). Detecting ADLs provides possibilities of improving the home life of elderly people as it can be applied to fall detection systems. This paper presents fall detection in elderly people based on radar image classification by examining their daily routine activities, using radar data that were previously collected for 99 volunteers. Machine learning techniques are used classify six human activities, namely walking, sitting, standing, picking up objects, drinking water and fall events. Different machine learning algorithms, such as random forest, K-nearest neighbours, support vector machine, long short-term memory, bi-directional long short-term memory and convolutional neural networks, were used for data classification. To obtain optimum results, we applied data processing techniques, such as principal component analysis and data augmentation, to the available radar images. The aim of this paper is to improve upon the results achieved using a publicly available dataset to further improve upon research of fall detection systems. It was found out that the best results were obtained using the $\mathrm{CNN}$ algorithm with principal component analysis and data augmentation together to obtain a result of $95.30 \%$ accuracy. The results also demonstrated that principal component analysis was most beneficial when the training data were expanded by augmentation of the available data. The results of our proposed approach, in comparison to the state of the art, have shown the highest accuracy.
\end{abstract}

Keywords: activity detection; machine learning; radar sensing; wireless sensing

\section{Introduction}

The growing numbers of elderly people present numerous challenges within the healthcare industry. These new challenges demand new methods which can be implemented in a cost-effective manner [1]. Assistive technology can serve to improve the care of elderly people. The healthcare industry is looking to technology to support growing needs, as demonstrated in [2-4]. Advances in sensor technology are currently being used to support the elderly population [5] and can be used to detect large-scale body movements such as walking, sitting down on a chair, standing up from a chair, picking up objects, fall events, etc.

The World Health Organization (WHO) has reported that $42 \%$ of people over 70 years old experience fall at least once a year [6], which can be caused by various hazards such as slippery floors and low lighting conditions, and the damage can be greater due to elderly 
people's bodies being fragile [7]. The risk of falling also increases with age. Falls in elderly people can cause more serious injuries than in younger people [8]. Conditions such as arthritis and visual impairment can increase the chance that an elderly person will fall [9]. Arthritis is a condition that causes pain in the joints [10]. This pain when moving could potentially result in elderly people falling as they struggle to move. Visual impairment can cause the elderly to struggle to see hazards and thus trip and fall. Conditions such as nocturia, which causes older people to wake up during the night with a need to go to the toilet, can pose a greater risk of falling as they try to manoeuvre around the home when tired and under poor lighting [8]. Falling can risk serious injury which can result in hospital visits or permanent disabilities [11]. This can lead to sufferers of falls to no longer be able to live independently for fear of further injury. Moreover, fall events are the second leading cause of accidental death [12].

In this context, various sensing technologies, such as wearable accelerometer devices, vibration sensing, visual sensing and radar-based sensing [13], have been used to monitor daily routine activities of elderly people. Wearable devices use accelerometers to be able to detect the movement of the person wearing the device and several factors must be considered, as elderly people may not wear the devices if they find them uncomfortable [14]. Additionally, elderly people may suffer from conditions that inhibit their memory. They may forget to wear the device at times, and it will no longer be able to sense the movements of that person. Vibration, visual sensing and radar-based sensing work without having any devices introduced to the body. These methods are classified as non-invasive sensing methods. Vibration sensing works using a microphone to record the vibrations on the floor as a person experiences a fall [15]. Visual sensing can be achieved by leveraging camera-based technology. Cameras can be used to record elderly people and frames can be analysed using machine learning techniques to assess if a fall has occurred. It also allows for caregivers to view the situation and decide if further care is required [16]. Cameras may not be ideal as some elderly people will not agree to be under constant surveillance. Radar-based sensing works by exploiting the Doppler signatures created on radar when a fall occurs [17]. This paper aims to implement different machine learning and data processing techniques to improve the existing results of an available dataset containing radar micro-Doppler spectrograms of human activities. The research detailed in this study focuses on the effectiveness of radar-based sensing in detecting activities of daily living. Machine learning (ML) algorithms are applied to a dataset to predict if these activities can be classified using artificial intelligence (AI). The machine learning algorithms used are random forest $(\mathrm{RF}), \mathrm{K}$-nearest neighbours $(\mathrm{KNN})$, support vector machine (SVM), long short-term memory (LSTM), bi-directional Long short-term memory (BiLSTM) and convolutional neural network (CNN).

The paper is organised into the following sections. Section 2 provides detailed related work within the field of radar-based motion detection. Section 3 presents the methodology of how the experimentation of this research has been achieved. Section 4 discusses the results obtained through the experimentation. Section 5 gives the conclusion.

\section{Related work}

The following section provides details with regard to state-of-the-art work in the field of human activity recognition using radar sensing technology in conjunction with machine learning methods that have been applied for classification purposes. The work of [18] used the same dataset used in this paper. The paper used SVM, KNN and GoogleNet algorithms to classify the data. The results produced using these algorithms had $78.25 \%$ accuracy for SVM, $77.15 \%$ accuracy for KNN and $74.70 \%$ accuracy for GoogleNet. The work of our paper seeks to improve on these results. The paper [19] included radar spectrograms of activities such as walking, falling, sitting down and bending down. The spectrograms were used for image classification and images were converted to greyscale for data preprocessing. The algorithms deep neural network (DNN) and SVM were applied to the pre-processed data. DNN achieved accuracy results of $87.00 \%$ while SVM achieved an 
accuracy score of $78.00 \%$. Research in [20] used spectrograms for image classification using greyscale. The images were then classified using the SVM algorithm. The authors developed sequential forward selection (SFS) for feature selection. The results of the classification achieved between $92.00 \%$ and $95.00 \%$ accuracy depending on the number of features used. The paper [21] included the algorithms stacked recurrent neural network (RNN) with LSTM and deep CNN to classify six human motions using radar Doppler images. The six motions were boxing, hand clapping, hand waving, piaffe, jogging and walking. The results achieved in these experiments showed that the stacked recurrent neural network (RNN) with LSTM achieved $92.65 \%$ accuracy, with the deep CNN achieving 82.33\% accuracy. Erol and Amin [22] used spectrograms and range maps of human motion and fused them together for classification. The spectrogram results are similar to the experiments conducted in our study. The study investigated five human motions, namely bending, falling, sitting, kneeling and walking. The spectogram experiment used obtained a result of $82.24 \%$ accuracy using KNN with PCA. With fusion methods of the spectrograms and range maps, we were able to achieve a result of $93.94 \%$ using KNN with PCA. The work of [23] used impulse-radio ultra-wideband (IR-UWB) radar to capture 12 kinds of motions. The data processing used KNN to define the features in the spectograms. Then, the power spectrum and Doppler shifts were extracted and sent to a CNN algorithm for classification. This was run using five-fold cross validation and achieved up to $98 \%$ for detecting the human motions. The study [24] used IR-UWB radar with CNN to be able to identify between falling and activities of daily living. This work used binary classification and focused on differentiating between the falling motion and any other type of activity around the home. The CNN algorithm was able to provide an accuracy score of $96.35 \%$. The authors of [25] made use of UWB radars to create a dataset that contained 10 subjects aged between 22 and 39 years old, performing 15 different activities. The data were collected while other people were still active in the building behind walls. This was to simulate a realistic care home environment where other residents reside in neighbouring apartments. The experiment achieved an accuracy score of $80 \%$ using the random forest algorithm. The work of [26] used a UWB radar with seven subjects carrying out four activities, namely walking, sitting, standing and simulated falling. The collected data were run using 10-fold cross validation and it was found that KNN performed the best with a result of $94.90 \%$ accuracy. The authors of [27] used UWB radars to collect data for binary classification of falling and non-falling events. Data were collected using 10 volunteers in three different locations within a proposed apartment. They validated their results by using the leave one subject out method and found that the achieved accuracy was $90.00 \%$ using a CNN-LSTM architecture deep learning model.

\section{Methodology}

In this section, we discuss our proposed methodology for fall detection in elderly people. This study makes use of radar micro-Doppler spectrograms of human activities. These data were taken from [18] and are publicly available The data were collected for 99 elderly people at nine different locations, namely the University of Glasgow, a north Glasgow elderly care home and Age UK West Cumbria. The dataset can be found at http:/ / researchdata.gla.ac.uk/848/, accessed on 20 April 2020. The dataset consists of six motions: walking, sitting down, standing up, picking up an object, drinking water and falling. The dataset of the radar spectrograms is in the format of 227 by 227-pixel PNG image files. There is a total of 1633 spectrograms in the dataset. Table 1 shows the breakdown of how many spectrograms are present for each motion. 
Table 1. Number of spectrograms per motion in the dataset.

\begin{tabular}{ll}
\hline Algorithm & Number of Samples \\
\hline Walking & 286 \\
\hline Sitting Down & 289 \\
\hline Standing Up & 287 \\
\hline Picking Up an Object & 287 \\
\hline Drinking Water & 286 \\
\hline Falling & 198 \\
\hline Total & 1633 \\
\hline
\end{tabular}

Figure 1 shows spectrogram examples of each activity. The Skimage Python package was used to convert the image files into data based on pixel information and store this data in Python arrays [28]. The image data array was in the shape of $[227,227,3]$. This represents the 227-pixel width and 227-pixel height of the PNG file. The 3 represents the red, green and blue (RGB) values of the pixels. The images were then converted into greyscale which removed the colour and changed the array shape to $[227,277,1]$. This array of data was saved in a CSV format for machine learning processing. The conversion to greyscale was used to reduce the dimensions. The RGB channels were thus converted to a single intensity value [29]. Greyscale images have been shown to have improved classification accuracy over coloured images [30] and this method has been used in various related works involving radar image classification [20,31]. The experiments conducted in this study used four different data processing methods. The first method used the raw greyscale pixel data only. The second method then applied principal component analysis (PCA) to the raw greyscale pixel data. PCA is a technique used to reduce dataset dimensions [32]. PCA finds common components of the data which can summarise the variation of the data [33]. The third method used data augmentation of the raw greyscale pixel data. Data augmentation is the process of increasing the training data by transforming an existing sample [34]. The original and transformed samples can then be part of the same dataset and thus the amount of training data is increased. The transformed samples must keep the variance intact that defines original sample labels within the dataset. PCA is applied in this study by using sklearn. Sklearn provides a function that can fit and transform the image data using PCA. PCA is configured to use all components of the data. Data augmentation in our study tripled the size of the dataset. The data augmentations contain the original greyscale images, blurred greyscale images and horizontally flipped greyscale images. Using blurred and flipped images increases the size of the dataset but these occurrences are unlikely to happen in real life systems. The reason this was carried out in this work is that it allows for more data to be produced while still maintaining some of the original features of the data without causing duplicates in the dataset. The fourth and final method used data augmentation and PCA combined. Figure 2 shows the process followed when creating the data augmentation dataset. Using these four data processing techniques, four different datasets were produced that could then have machine learning and applied and results analysed. The datasets were divided into $90 \%$ for training data and $10 \%$ for testing data. This was achieved by using the train_test_split function included in the sklearn python package. The function parameters were used to define the percentage of testing data and the rest was used as training data. The data were shuffled but the random seed parameter was always the same to ensure all algorithms used the same samples as testing and training data. This allowed for the results to reflect the model generalisation of the algorithms due to the specific training data remaining consistent and ensuring that the testing samples were the same unseen samples in every experiment conducted. 


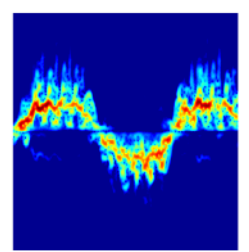

Walking

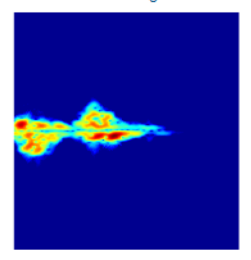

Picking up Object

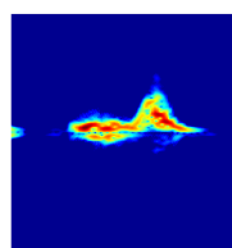

Sitting Down

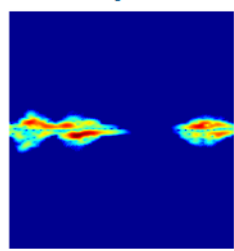

Drinking Water

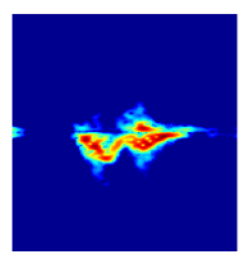

Standing Up

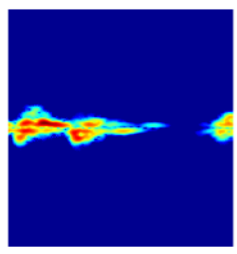

Falling Down

Figure 1. Raw spectrogram examples of each activity [18].

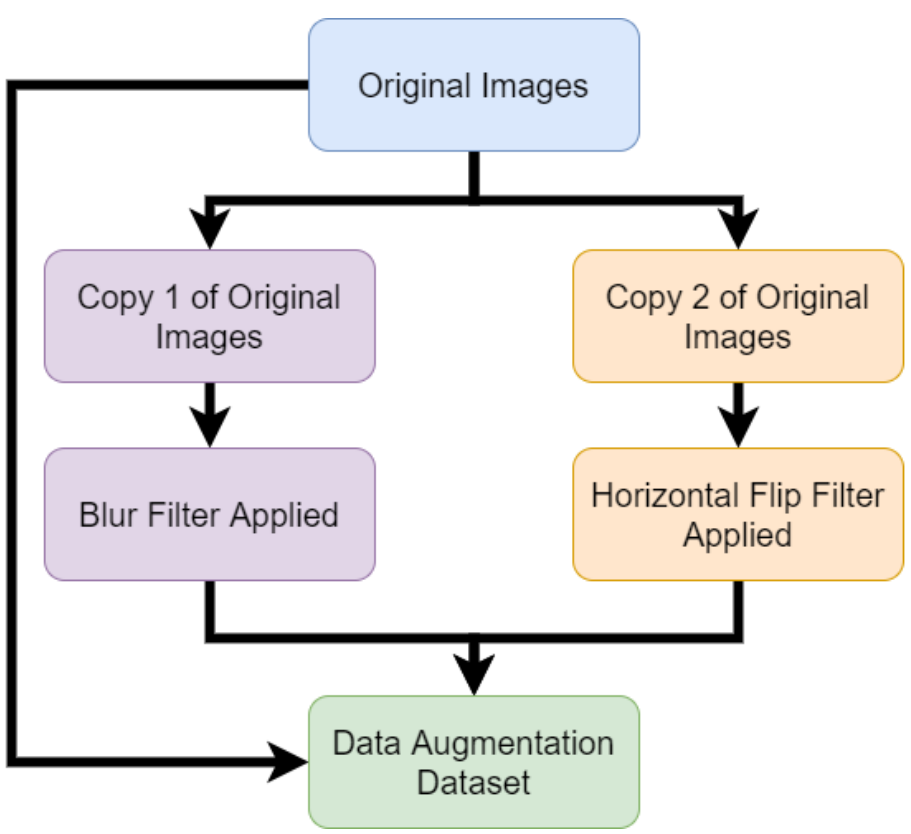

Figure 2. Flow graph of how data augmentation dataset was created.

\section{Machine Learning}

We have used different machine learning algorithm, such as random forest, KNN, SVM, LTSM, BiLTSM and CNN. The Scikit python package was used to implement random forest, KNN and SVM. Scikit is widely used in industry and research within the field of data science [35]. To implement the neural network algorithms LTSM, BiLTSM and CNN, the Tensorflow Python package was used. Tensorflow was used for this research as it is open source and can be used with a graphics processing unit (GPU) [36]. The use of a GPU allows for neural network processes to run faster than if the processes were to be run on a CPU.

The random forest algorithm is a collection of decision trees. Each individual decision tree will make a decision on which classification to assign to new data based on key features learnt in the training phase. The classification each tree reaches is considered a vote. The final classification decided by the random forest algorithm is the ensemble classification of the combined decision trees [37]. In these experiments, the random forest was configured to use 200 trees in the forest. This number of trees resulted in the best results in the initial experimentation. 
The KNN algorithm simply looks to compare training data to new unseen data [38]. The features of the training data are assigned $\mathrm{K}$ values. The features of the new data are then assigned a $\mathrm{K}$ value which best matches the features of the training data [39]. The parameter used for KNN in this study was set to use 10 neighbours.

SVM aims to create boundaries between each classification. These boundaries are known as hyper planes. The hyper plane is positioned as far as possible from the closest data points of the classes present in the data. These points are known as the support vectors [40]. The hyper planes are used to divide the support vectors into the different categories. The training data features are used to place the hyper planes. Then, the features of the new data are used to place the new data between the hyper planes and provide classification [41]. The SVM parameters included the gamma set to auto and the use of the RBF kernel in these experiments.

The LSTM deep learning algorithm is an extension of a recurring neural network (RNN). A recurring neural network is a type of neural network which models the dynamic behaviour of sequences of data between nodes of the neural network. LSTM expands on this concept by adding three different gates to the nodes. One gate is used to decide if the current state should be forgotten. Another gate is used to control if input should be read or not and the final gate decides if the state should be added to the node output. These gates allow for LSTM to decide if the sequence of data is relevant to the output of the node [42]. In this experiment, LSTM was used with 50 nodes, also known as units. The learning rate was set to $1 \times 10^{-6}$ using ReLU activation and ran for 200 epochs. Figure 3 shows a visual representation of how LSTM works.

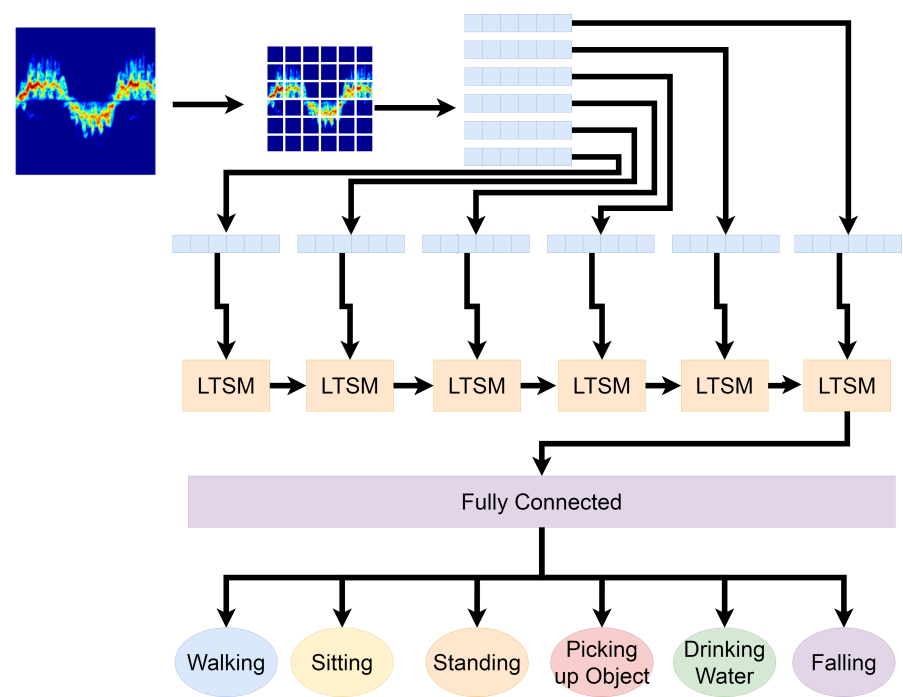

Figure 3. Visual representation of how LSTM works.

The bi-directional long short-term algorithm is an extension of the LSTM deep learning algorithm. Where LSTM only makes use of the past behaviours of data sequences, the bidirectional long short-term algorithm can look at data in two directions. The two directions look at past and future data in a sequence. This is achieved by using two LSTM networks. One LSTM network, the forward LSTM network, can review past data sequences and the backward LSTM network can review future data sequences [43]. BiLSTM was configured to use the same parameters as the LSTM set up. Figure 4 shows a visual representation of how BiLSTM works.

The CNN algorithm is an emerging technology which is a powerful solution for image classification problems which were initially thought to require human intelligence $[44,45]$. The CNN algorithm is made up of densely connected layers that take the activations of all the previous layers as input. The layers produce feature maps from this input which are known as growth rates [46]. The convolution neural network architecture used in this study consisted of multiple convolutions and pooling layers followed by multiple connected 
layers (ReLU) and finally a softmax layer for creating the output classes. The convolution and pooling layers were used to capture the features of the images. The weight and bias of the features were parameters of the $\mathrm{CNN}$ which were configured automatically during the training process [47]. Figure 5 displays the $\mathrm{CNN}$ model used in this experiment.

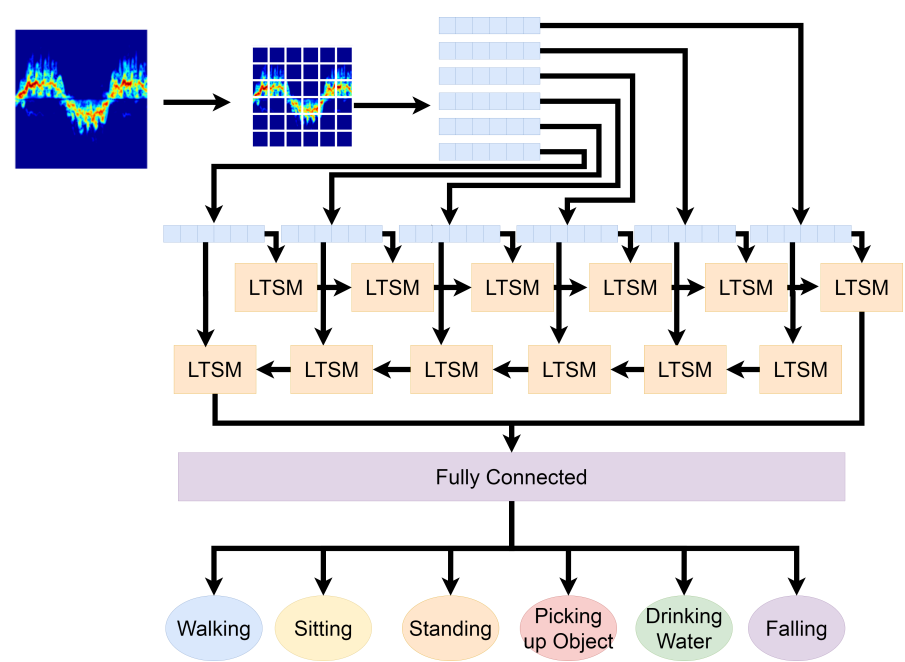

Figure 4. Visual representation of how BiLSTM works.

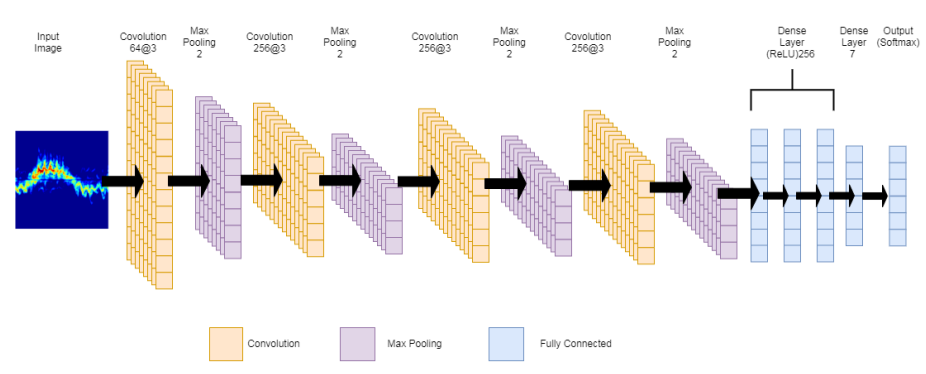

Figure 5. Visual representation of CNN model used in experiment.

\section{Results and Discussion}

This section presents the weighted average results of each of the classifications carried out in each experiment. Each experiment includes the weighted average accuracy in bar chart format and then the weighted average accuracy, precision, recall and F1-score.

\subsection{Original Dataset}

\subsubsection{Raw Images}

The accuracy results of each machine learning algorithm using just the raw images are visually displayed in bar chart format in Figure 6.

The results of the machine learning algorithms show that KNN has superior performance using the raw image data. KNN has an accuracy of $90.85 \%$. Although KNN performs the best, all the algorithms can classify the majority of the images correctly. These results show that the machine learning algorithms applied to the image data are able to identify the differences in the image data, with $\mathrm{KNN}$ being able to distinguish the differences at a higher rate than the other algorithms. The other algorithms were still able to recognise differences in the images, however, $\mathrm{CNN}$ was the second-best performer with $85.97 \%$ accuracy. SVM is the weakest performer out of all algorithms but still achieved $71.34 \%$ accuracy.

Table 2 shows the accuracy and the average precision, recall and F1-score of the machine learning algorithms using the raw images. 


\subsubsection{Principal Component Analysis}

The accuracy results of each machine learning algorithm using PCA are visually displayed in bar chart format in Figure 7.

The results of the PCA described a drop in comparison to using the raw image data. KNN maintained its status as being the highest performer of the machine learning algorithms but it dropped by $13.42 \%$ in accuracy from $90.85 \%$ when using raw data to $77.43 \%$ when using PCA. SVM had the smallest drop in performance compared to the other algorithms when using PCA with a drop of $1.22 \%$, from $71.34 \%$ down to $70.12 \%$. These results show that using PCA decreases the accuracy compared to the results of using raw data. The deep learning algorithms were still ultimately able to differentiate between human motions using PCA despite the drop in accuracy.

Table 2 shows the accuracy and the average precision, recall and F1-score of the machine learning algorithms and Table 3 shows the average precision, recall and F1-score of the machine learning algorithms using PCA for the image data.

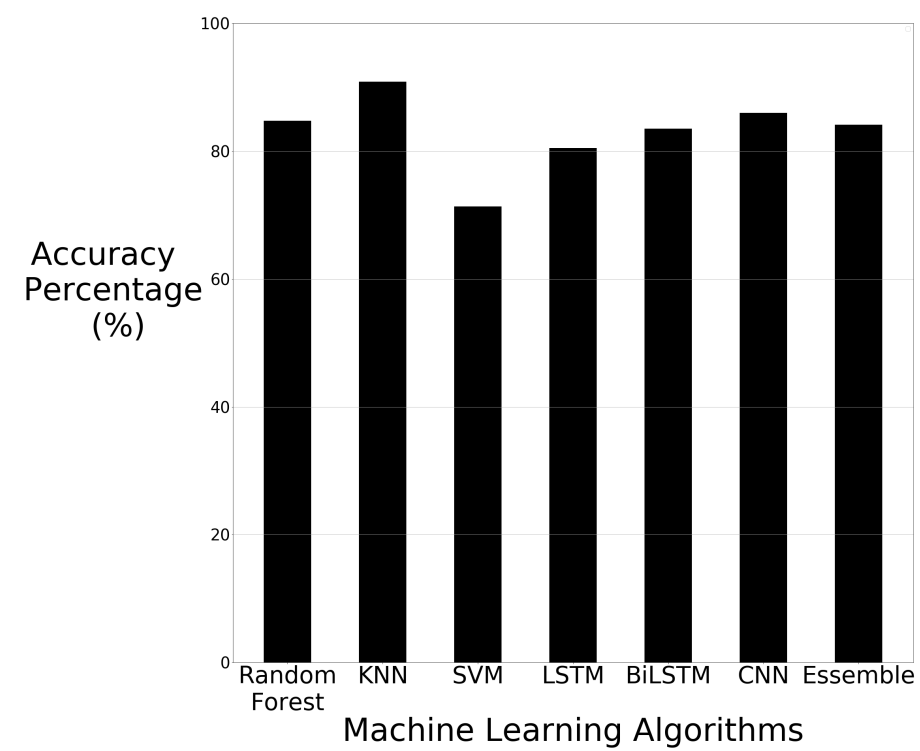

Figure 6. Machine learning results using raw image data.

Table 2. Machine learning results using raw image data.

\begin{tabular}{ccccc}
\hline Algorithm & Accuracy & Precision & Recall & F1-Score \\
\hline Random Forest & $84.75 \%$ & 0.86 & 0.85 & 0.85 \\
\hline KNN & $90.85 \%$ & 0.91 & 0.91 & 0.91 \\
\hline SVM & $71.34 \%$ & 0.74 & 0.71 & 0.71 \\
\hline LSTM & $80.48 \%$ & 0.83 & 0.80 & 0.80 \\
\hline BiLSTM & $83.53 \%$ & 0.87 & 0.84 & 0.84 \\
\hline CNN & $85.97 \%$ & 0.86 & 0.86 & 0.86 \\
\hline
\end{tabular}


Table 3. Machine learning results using raw image data with PCA.

\begin{tabular}{ccccc}
\hline Algorithm & Accuracy & Precision & Recall & F1-Score \\
\hline Random Forest + PCA & $76.21 \%$ & 0.78 & 0.76 & 0.76 \\
\hline KNN + PCA & $77.43 \%$ & 0.78 & 0.77 & 0.77 \\
\hline SVM + PCA & $70.12 \%$ & 0.71 & 0.70 & 0.70 \\
\hline LSTM + PCA & $75.00 \%$ & 0.76 & 0.75 & 0.75 \\
\hline BiLSTM + PCA & $73.78 \%$ & 0.74 & 0.74 & 0.74 \\
\hline CNN + PCA & $76.82 \%$ & 0.78 & 0.77 & 0.77 \\
\hline
\end{tabular}

\subsection{Data Augmentation}

\subsubsection{Raw Images}

The accuracy results of the machine learning algorithms using data augmentation is visually displayed in bar chart format in Figure 8.

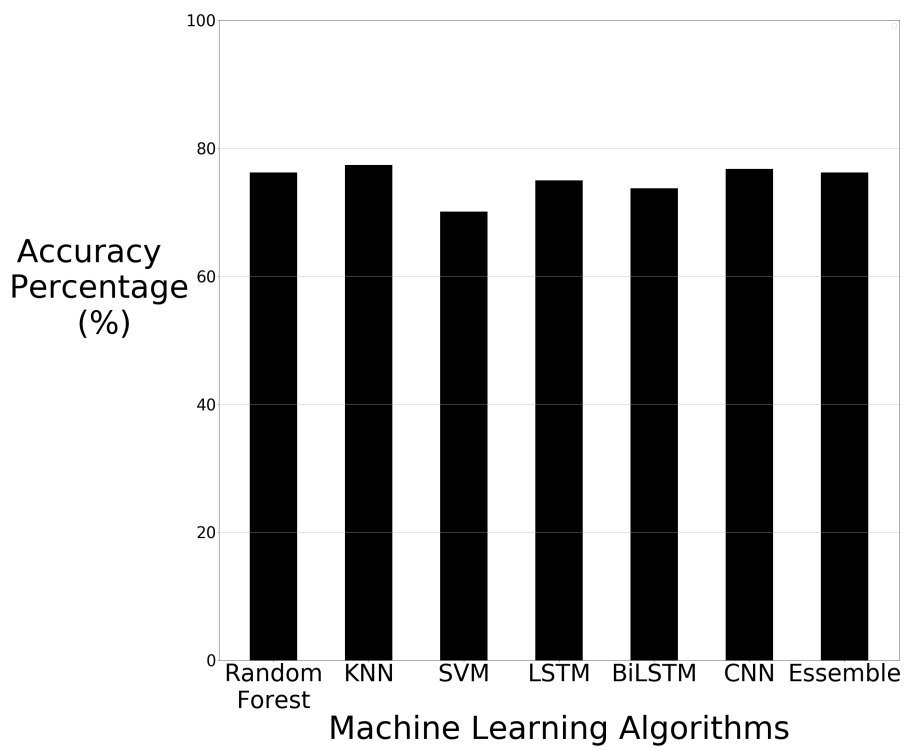

Figure 7. Machine learning results using raw image data with PCA.

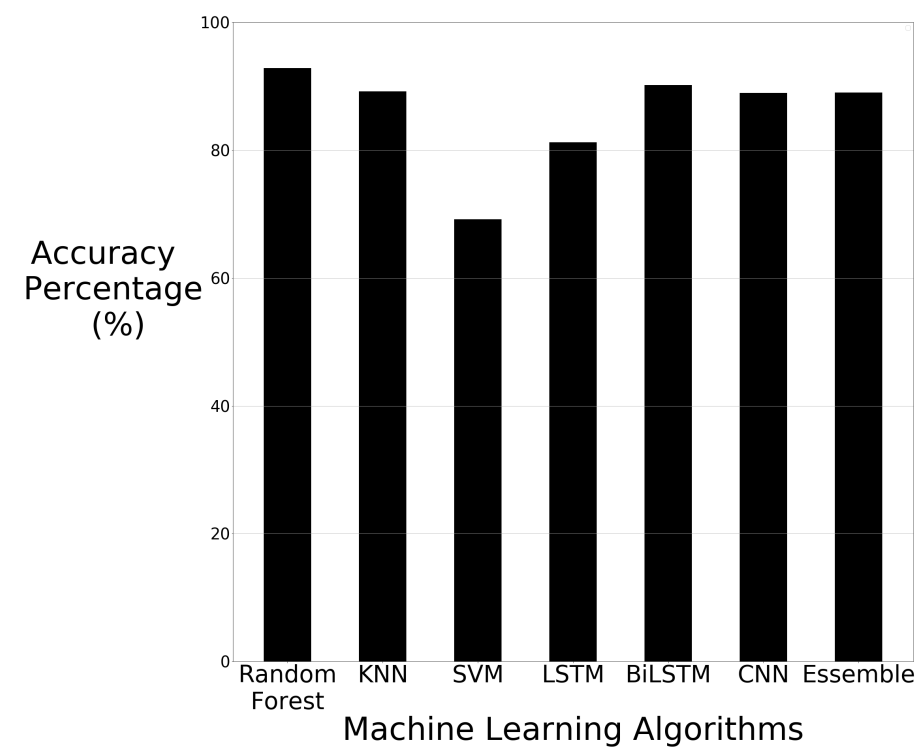

Figure 8. Machine learning results using data augmentation. 
The results of using data augmentation have shown that random forest increased in accuracy with the benefits of having more training data in comparison to only using raw images with no data augmentation. The accuracy of random forest increased by $8.1 \%$, from $84.75 \%$ to $92.85 \%$. KNN and SVM showed decreased accuracy scores with the added training data. The accuracy of KNN dropped by $1.67 \%$, from $90.85 \%$ to $89.18 \%$. The drop in accuracy with SVM was $2.16 \%$, from $71.34 \%$ down to $69.18 \%$. SVM still remains the lowest performer out of all the machine learning algorithms, whereas random forest outperformed $\mathrm{KNN}$ and was the only algorithm that benefited from the larger dataset. The BiLSTM algorithm showed increased accuracy by $6.67 \%$, from $83.53 \%$ to $90.20 \%$, making it the second best-performing algorithm. The CNN algorithm had an accuracy of $88.97 \%$ when using the data augmentation dataset. It had an accuracy increase of $3.00 \%$ compared to using the raw image dataset. The LSTM did improve slightly when using the data augmentation dataset with an increase of $0.74 \%$, from $80.48 \%$ to $81.22 \%$. Table 4 shows the accuracy and the average Precision, recall and F1-score of the machine learning algorithms using data augmentation techniques.

Table 4. Machine learning results using data augmentation.

\begin{tabular}{ccccc}
\hline Algorithm & Accuracy & Precision & Recall & F1-Score \\
\hline Random Forest & $92.85 \%$ & 0.93 & 0.93 & 0.93 \\
\hline KNN & $89.18 \%$ & 0.89 & 0.89 & 0.89 \\
\hline SVM & $69.18 \%$ & 0.70 & 0.69 & 0.69 \\
\hline LSTM & $81.22 \%$ & 0.83 & 0.81 & 0.81 \\
\hline BiLSTM & $90.20 \%$ & 0.90 & 0.90 & 0.90 \\
\hline CNN & $88.97 \%$ & 0.91 & 0.89 & 0.89 \\
\hline
\end{tabular}

\subsubsection{Principal Component Analysis}

The accuracy results of the machine learning algorithms using data augmentation with PCA are displayed in Figure 9.

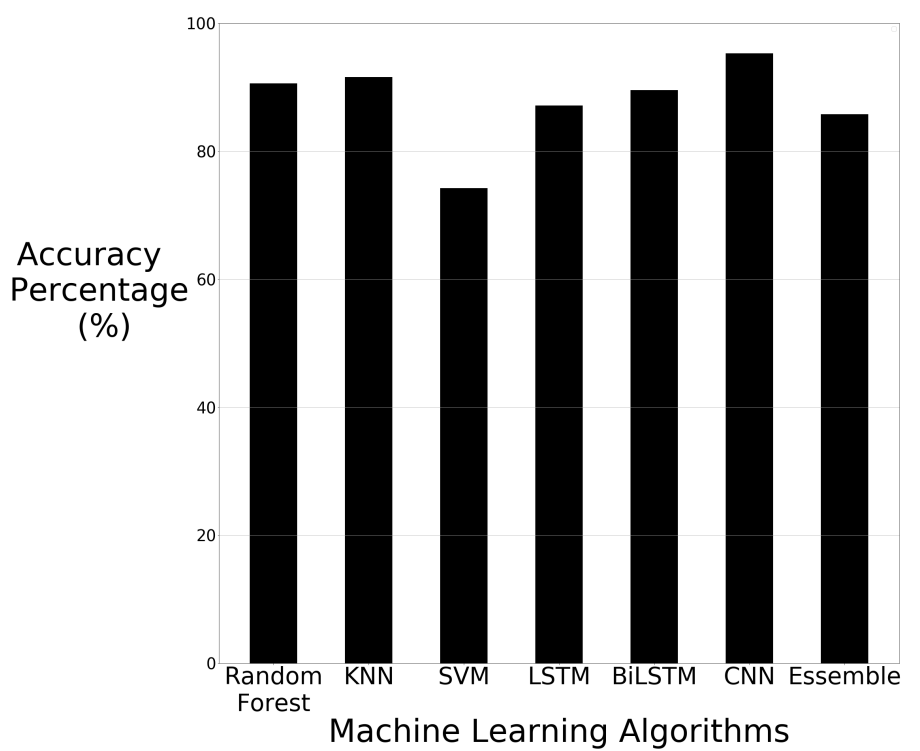

Figure 9. Machine learning results using data augmentation with PCA.

The machine learning results using PCA and data augmentation have shown that KNN, SVM, LTSM and CNN improved when using PCA and data augmentation in comparison to just using data augmentation. The KNN results increased from $89.18 \%$ to $91.63 \%$ 
compared to using only data augmentation techniques. SVM results increased from $69.18 \%$ to $74.28 \%$ compared to using only data augmentation techniques. LSTM showed an increased accuracy result from $81.22 \%$ up to $87.14 \%$ and CNN showed increased accuracy from $88.97 \%$ up to $95.30 \%$. The result from $\mathrm{CNN}$ is the highest achieved result in all experiments undertaken throughout this study.

When the KNN, SVM, LTSM and CNN algorithms were applied to the PCA dataset without data augmentation, the results decreased in performance. These results show that for KNN, SVM, LTSM and CNN, the additional training data help to improve the performance. The random forest and BiLTSM algorithms showed a decrease in performance when using PCA and data augmentation in comparison to only using data augmentation. The accuracy only fell by $2.24 \%$, from $92.85 \%$ down to $90.61 \%$, for random forest and BiLTSM fell from $90.20 \%$ to $89.59 \%$. This drop is significantly less severe compared to the drop observed in the results of random forest and $\mathrm{CNN}$ when using raw images to then using the raw images with PCA. Random forest dropped in accuracy by $8.54 \%$ and BiLTSM dropped in accuracy by $9.75 \%$. This shows that PCA performs better in most of the machine learning algorithms when the amount of training data is increased, with the exception being the random forest and $\mathrm{CNN}$ algorithms. Table 5 shows the accuracy and the average Precision, recall and F1-score of the machine learning algorithms using data augmentation techniques paired with PCA.

Table 5. Machine learning results using data augmentation with PCA.

\begin{tabular}{ccccc}
\hline Algorithm & Accuracy & Precision & Recall & F1-Score \\
\hline Random Forest + PCA & $90.61 \%$ & 0.91 & 0.91 & 0.91 \\
\hline KNN + PCA & $91.63 \%$ & 0.92 & 0.92 & 0.92 \\
\hline SVM + PCA & $74.28 \%$ & 0.75 & 0.74 & 0.74 \\
\hline LSTM + PCA & $87.14 \%$ & 0.88 & 0.87 & 0.87 \\
\hline BiLSTM + PCA & $89.59 \%$ & 0.90 & 0.90 & 0.90 \\
\hline CNN + PCA & $95.30 \%$ & 0.95 & 0.95 & 0.95 \\
\hline
\end{tabular}

\subsection{Ablation Studies}

This section details ablation. Ablation is the process of removing activities within the dataset and an analysis of how the reduced number of classifications compares to results achieved using the complete dataset. For this section, the best performing dataset when using data augmentation and PCA was reduced from six activities down to three. The three activities used were walking, sitting and standing. Figure 10 shows the accuracy of the comparison of each algorithm between three and six activities. Most of the algorithms achieved higher results when using fewer classifications, however, KNN and CNN algorithms performed worse with three activities. The difference with KNN was negligible and the difference in performance with $\mathrm{CNN}$ was likely due to the reduced amount of training data. In experiments using six activities, it was observed that $\mathrm{CNN}$ performs better with more training data. 


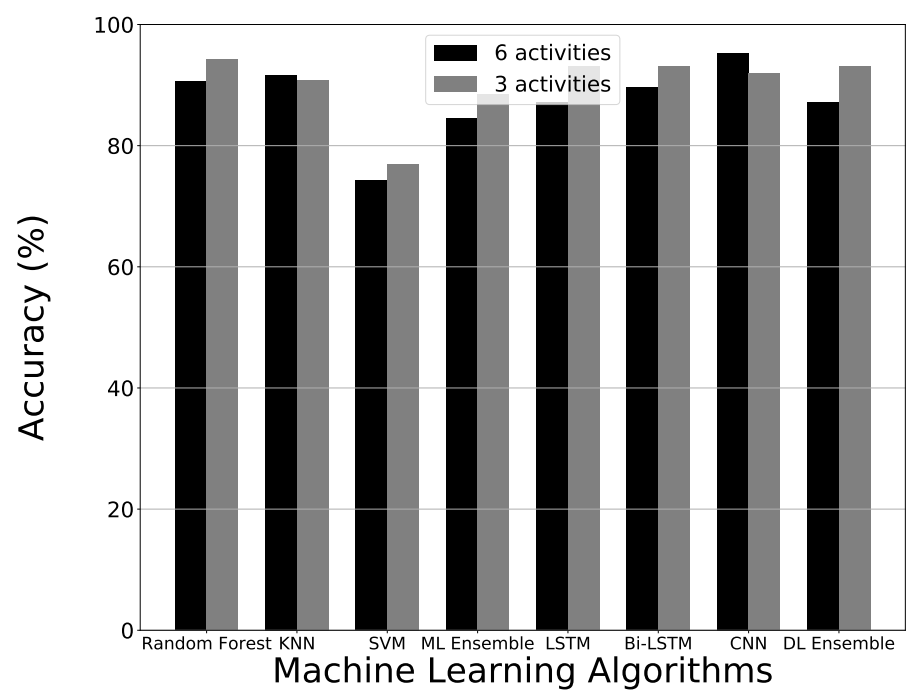

Figure 10. Ablation study results using data augmentation paired with PCA.

\section{Comparison to State-of-the-Art Approaches}

The results of our proposed approach can be directly compared to the results of [18], as this work used the same dataset used in the experiments of this study. The authors of [18] used SVM and KNN algorithms and these algorithms achieved an accuracy score of $78.25 \%$ and $77.15 \%$, respectively. SVM, in the results presented in this paper, achieved the highest result of $74.28 \%$, which was lower than the work shown in [18]. However, we were able to improve the KNN results in our work. In our experimentation, KNN achieved its highest result of $91.63 \%$ when using data augmentation paired with PCA. The data processing techniques used in this study have allowed for the KNN algorithm to improve the accuracy results by nearly 15\%. The work conducted in this study even expanded on the improvements in accuracy using the dataset with the use of CNN. The accuracy score of 95.30\% achieved when using CNN with data augmentation and PCA was the best accuracy score achieved in this work, showing an accuracy score increase of around $17 \%$ from the best accuracy score achieved by [18] when using the KNN algorithm.

\section{Conclusions}

This paper presented results obtained when machine and deep learning techniques were applied to a radar dataset that was previously generated containing radar microDoppler spectrograms for various human activities. The research work has shown that using PCA paired with augmentation of the available data achieved the best results of $95.30 \%$ using the deep learning algorithms, namely CNN. These results have outperformed the state-of-the-art approaches. The results also highlighted how PCA can achieve better results when the training data are expanded using data augmentation. Without data augmentation, it was found that PCA had a negative effect on the results. Although the highest machine learning result was obtained using random forest without PCA, the rest of the machine learning algorithms displayed higher results when PCA was used with data augmentation. The results of this paper have shown that AI can identify the different activities of daily living that are detected by radar sensing by performing image classification on acquired micro-Doppler signatures. Future work of this research can seek to implement systems that make use of the data processing and deep learning techniques presented in the findings of this paper to improve the lives of elderly people. 
Author Contributions: Conceptualization, W.T., K.D., S.A.S., A.H., Q.H.A., M.A.I.; methodology, W.T., S.A.S., K.D.; software, W.T., S.A.S., K.D.; validation, W.T., S.A.S., K.D.; formal analysis, W.T., K.D., S.A.S., A.H.; investigation, W.T., S.A.S., K.D.; resources, writing, review and editing, W.T., S.A.S., K.D., A.H., Q.H.A., M.I.A.; funding acquisition, Q.H.A., M.A.I. All authors have read and agreed to the published version of the manuscript.

Funding: William Taylor's studentship is funded by CENSIS UK through Scottish funding council in collaboration with British Telecom. This work is supported in parts by EPSRC DTG EP/N509668/1 Eng, EP/T021020/1 and EP/T021063/1.

Institutional Review Board Statement: Not applicable.

Informed Consent Statement: Not applicable.

Data Availability Statement: The dataset can be found at http://researchdata.gla.ac.uk/848/, accessed on 20 April 2021.

Acknowledgments: William Taylor's studentship is funded by CENSIS UK through the Scottish Funding Council in collaboration with British Telecom. This work is supported in part by EPSRC $\mathrm{EP} / \mathrm{T} 021020 / 1$ and EP/T021063/1.

Conflicts of Interest: The authors declare no conflict of interest.

\section{References}

1. Woll, A.; Bratteteig, T. A trajectory for technology-supported elderly care work. Comput. Support. Coop. Work. (CSCW) 2019, 28, 127-168. [CrossRef]

2. Yang, X.; Ren, X.; Chen, M.; Wang, L.; Ding, Y. Human Posture Recognition in Intelligent Healthcare. J. Phys. Conf. Ser. 2020, 1437, 012014. [CrossRef]

3. Abbasi, Q.H.; Rehman, M.U.; Qaraqe, K.; Alomainy, A. Advances in Body-Centric Wireless Communication: Applications and State-of-the-Art; Institution of Engineering and Technology: London, UK, 2016; ISBN 9781849199896.

4. Taylor, W.; Shah, S.A.; Dashtipour, K.; Zahid, A.; Abbasi, Q.H.; Imran, M.A. An Intelligent Non-Invasive Real-Time Human Activity Recognition System for Next-Generation Healthcare. Sensors 2020, 20, 2653. [CrossRef]

5. Grossi, G.; Lanzarotti, R.; Napoletano, P.; Noceti, N.; Odone, F. Positive technology for elderly well-being: A review. Pattern Recognit. Lett. 2019, 137, 61-70. [CrossRef]

6. De Miguel, K.; Brunete, A.; Hernando, M.; Gambao, E. Home camera-based fall detection system for the elderly. Sensors 2017, 17, 2864. [CrossRef]

7. Yang, L.; Ren, Y.; Zhang, W. 3D depth image analysis for indoor fall detection of elderly people. Digit. Commun. Netw. 2016, 2, 24-34. [CrossRef]

8. Taramasco, C.; Rodenas, T.; Martinez, F.; Fuentes, P.; Munoz, R.; Olivares, R.; De Albuquerque, V.H.C.; Demongeot, J. A novel monitoring system for fall detection in older people. IEEE Access 2018, 6, 43563-43574. [CrossRef]

9. Tsinganos, P.; Skodras, A. A smartphone-based fall detection system for the elderly. In Proceedings of the 10th International Symposium on Image and Signal Processing and Analysis, Ljubljana, Slovenia, 18-20 September 2017 ; pp. 53-58.

10. Ou, Y.; Ambalathankandy, P.; Shimada, T.; Kamishima, T.; Ikebe, M. Automatic Radiographic Quantification of Joint Space Narrowing Progression in Rheumatoid Arthritis Using POC. In Proceedings of the 2019 IEEE 16th International Symposium on Biomedical Imaging (ISBI 2019), Venice, Italy, 8-11 April 2019; pp. 1183-1187.

11. Hussain, F.; Umair, M.B.; Ehatisham-ul Haq, M.; Pires, I.M.; Valente, T.; Garcia, N.M.; Pombo, N. An Efficient Machine Learning-based Elderly Fall Detection Algorithm. arXiv 2019, arXiv:1911.11976.

12. Santiago, J.; Cotto, E.; Jaimes, L.G.; Vergara-Laurens, I. Fall detection system for the elderly. In Proceedings of the 2017 IEEE 7th Annual Computing and Communication Workshop and Conference (CCWC), Las Vegas, NV, USA, 9-11 January 2017 ; pp. 1-4.

13. Sehairi, K.; Chouireb, F.; Meunier, J. Elderly fall detection system based on multiple shape features and motion analysis. In Proceedings of the 2018 International Conference on Intelligent Systems and Computer Vision (ISCV), Fez, Morocco, 2-4 April 2018; pp. 1-8.

14. Daher, M.; Diab, A.; El Najjar, M.E.B.; Khalil, M.A.; Charpillet, F. Elder tracking and fall detection system using smart tiles. IEEE Sens. J. 2016, 17, 469-479. [CrossRef]

15. Okumura, N.; Yamanoi, Y.; Kato, R.; Yamamura, O. Fall detection and walking estimation using floor vibration for solitary elderly people. In Proceedings of the 2019 IEEE International Conference on Systems, Man and Cybernetics (SMC), Bari, Italy, 6-9 October 2019; pp. 1437-1442.

16. Soni, P.K.; Choudhary, A. Automated Fall Detection From a Camera Using Support Vector Machine. In Proceedings of the 2019 Second International Conference on Advanced Computational and Communication Paradigms (ICACCP), Gangtok, India, 25-28 February 2019; pp. 1-6.

17. Erol, B.; Amin, M.G.; Boashash, B. Range-Doppler radar sensor fusion for fall detection. In Proceedings of the 2017 IEEE Radar Conference (RadarConf), Seattle, WA, USA, 8-12 May 2017; pp. 0819-0824. 
18. Shah, S.A.; Fioranelli, F. Human activity recognition: Preliminary results for dataset portability using FMCW Radar. In Proceedings of the 2019 International Radar Conference (RADAR), Toulon, France, 23-27 September 2019; pp. 1-4.

19. Jokanovic, B.; Amin, M.; Ahmad, F. Radar fall motion detection using deep learning. In Proceedings of the 2016 IEEE radar conference (RadarConf), Philadelphia, PA, USA, 1-6 May 2016; pp. 1-6.

20. Erol, B.; Amin, M.G.; Boashash, B.; Ahmad, F.; Zhang, Y.D. Wideband radar based fall motion detection for a generic elderly. In Proceedings of the 2016 50th Asilomar Conference on Signals, Systems and Computers, Pacific Grove, CA, USA, 6-9 November 2016; pp. 1768-1772.

21. Wang, M.; Zhang, Y.D.; Cui, G. Human motion recognition exploiting radar with stacked recurrent neural network. Digit. Signal Process. 2019, 87, 125-131. [CrossRef]

22. Erol, B.; Amin, M. Generalized pca fusion for improved radar human motion recognition. In Proceedings of the 2019 IEEE Radar Conference (RadarConf), Boston, MA, USA, 22-26 April 2019; pp. 1-5.

23. Qi, R.; Li, X.; Zhang, Y.; Li, Y. Multi-Classification Algorithm for Human Motion Recognition Based on IR-UWB Radar. IEEE Sens. J. 2020, 20, 12848-12858. [CrossRef]

24. Han, T.; Kang, W.; Choi, G. IR-UWB Sensor Based Fall Detection Method Using CNN Algorithm. Sensors 2020, $20,5948$. [CrossRef] [PubMed]

25. Bouchard, K.; Maitre, J.; Bertuglia, C.; Gaboury, S. Activity recognition in smart homes using UWB radars. Procedia Comput. Sci. 2020, 170, 10-17. [CrossRef]

26. Chowdhury, A.; Das, T.; Rani, S.; Khasnobish, A.; Chakravarty, T. Activity recognition using ultra wide band range-time scan. In Proceedings of the 2020 28th European Signal Processing Conference (EUSIPCO), Amsterdam, The Netherlands, 24-28 August 2020; pp. 1338-1342.

27. Maitre, J.; Bouchard, K.; Bertuglia, C.; Gaboury, S. Recognizing activities of daily living from UWB radars and deep learning. Expert Syst. Appl. 2021, 164, 113994. [CrossRef]

28. Van der Walt, S.; Schönberger, J.L.; Nunez-Iglesias, J.; Boulogne, F.; Warner, J.D.; Yager, N.; Gouillart, E.; Yu, T.; The Scikit-Image Contributors. Scikit-image: Image processing in Python. PeerJ 2014, 2, e453. [CrossRef]

29. Güneş, A.; Kalkan, H.; Durmuş, E. Optimizing the color-to-grayscale conversion for image classification. Signal Image Video Process. 2016, 10, 853-860. [CrossRef]

30. Bui, H.M.; Lech, M.; Cheng, E.; Neville, K.; Burnett, I.S. Using grayscale images for object recognition with convolutional-recursive neural network. In Proceedings of the 2016 IEEE Sixth International Conference on Communications and Electronics (ICCE), Ha-Long City, Quang Ninh, Vietnam, 27-29 July 2016; pp. 321-325.

31. Sakamoto, T. Personal identification using ultrawideband radar measurement of walking and sitting motions and a convolutional neural network. arXiv 2020, arXiv:2008.02182.

32. Jolliffe, I.T.; Cadima, J. Principal component analysis: A review and recent developments. Philos. Trans. R. Soc. A Math. Phys. Eng. Sci. 2016, 374, 20150202. [CrossRef]

33. Aït-Sahalia, Y.; Xiu, D. Principal component analysis of high-frequency data. J. Am. Stat. Assoc. 2019, 114, 287-303. [CrossRef]

34. Xie, Q.; Dai, Z.; Hovy, E.; Luong, M.T.; Le, Q.V. Unsupervised data augmentation. arXiv 2019, arXiv:1904.12848.

35. Hao, J.; Ho, T.K. Machine Learning Made Easy: A Review of Scikit-learn Package in Python Programming Language. J. Educ. Behav. Stat. 2019, 44, 348-361. [CrossRef]

36. Vishal, B.L.; Pragathi, M.; Amulya, P.K.; Kumar, N.S. Image Classification Using Neural Networks and Tensor-Flow; The Mattingley Publishing Co., Inc.: Oakland, CA, USA, 2020 .

37. Shaikhina, T.; Lowe, D.; Daga, S.; Briggs, D.; Higgins, R.; Khovanova, N. Decision tree and random forest models for outcome prediction in antibody incompatible kidney transplantation. Biomed. Signal Process. Control 2019, 52, 456-462. [CrossRef]

38. Saçlı, B.; Aydınalp, C.; Cansız, G.; Joof, S.; Yilmaz, T.; Çayören, M.; Önal, B.; Akduman, I. Microwave dielectric property based classification of renal calculi: Application of a kNN algorithm. Comput. Biol. Med. 2019, 112, 103366. [CrossRef] [PubMed]

39. Li, K.; Gu, Y.; Zhang, P.; An, W.; Li, W. Research on KNN Algorithm in Malicious PDF Files Classification under Adversarial Environment. In Proceedings of the 2019 4th International Conference on Big Data and Computing, Guangzhou, China, 10-12 May 2019; pp. 156-159.

40. Huang, S.; Cai, N.; Pacheco, P.P.; Narrandes, S.; Wang, Y.; Xu, W. Applications of support vector machine (SVM) learning in cancer genomics. Cancer Genom. Proteom. 2018, 15, 41-51.

41. Jain, M.; Narayan, S.; Balaji, P.; Bhowmick, A.; Muthu, R.K. Speech emotion recognition using support vector machine. arXiv 2020, arXiv:2002.07590.

42. Wang, J.; Yang, Y.; Mao, J.; Huang, Z.; Huang, C.; Xu, W. Cnn-rnn: A unified framework for multi-label image classification. In Proceedings of the IEEE Conference on Computer Vision and Pattern Recognition, Las Vegas, NV, USA, 27-30 June 2016; pp. 2285-2294.

43. Wang, S.; Wang, X.; Wang, S.; Wang, D. Bi-directional long short-term memory method based on attention mechanism and rolling update for short-term load forecasting. Int. J. Electr. Power Energy Syst. 2019, 109, 470-479. [CrossRef]

44. Chang, P.D.; Kuoy, E.; Grinband, J.; Weinberg, B.D.; Thompson, M.; Homo, R.; Chen, J.; Abcede, H.; Shafie, M.; Sugrue, L.; et al. Hybrid 3D/2D convolutional neural network for hemorrhage evaluation on head CT. Am. J. Neuroradiol. 2018, 39, 1609-1616. [CrossRef] [PubMed] 
45. Ren, A.; Zahid, A.; Zoha, A.; Shah, S.A.; Imran, M.A.; Alomainy, A.; Abbasi, Q.H. Machine learning driven approach towards the quality assessment of fresh fruits using non-invasive sensing. IEEE Sens. J. 2019, 20, 2075-2083. [CrossRef]

46. Elbayad, M.; Besacier, L.; Verbeek, J. Pervasive attention: $2 \mathrm{~d}$ convolutional neural networks for sequence-to-sequence prediction. arXiv 2018, arXiv:1808.03867.

47. Azamfar, M.; Singh, J.; Bravo-Imaz, I.; Lee, J. Multisensor data fusion for gearbox fault diagnosis using 2-D convolutional neural network and motor current signature analysis. Mech. Syst. Signal Process. 2020, 144, 106861. [CrossRef] 